\title{
Effects of aromatic chemistry-turbulence interactions on soot formation in a turbulent non-premixed flame
}

\author{
Y. Xuan ${ }^{1}$, G. Blanquart ${ }^{2}$ \\ ${ }^{1}$ Graduate Aerospace Laboratories, ${ }^{2}$ Department of Mechanical Engineering, \\ California Institute of Technology, Pasadena, USA
}

Contact information of the corresponding author

Yuan Xuan

Mailing address: 1200 E. California Blvd., MC 105-50

Pasadena, CA 91125, USA

Fax: (626) 568-2719

Email: yxuan@caltech.edu

Submission colloquium: Soot, PAH \& Other Large Molecules

Length of the paper: 6198 words determined by Method 2

\author{
Main text: 4272 words \\ References:874 words \\ Fig. 1: 71 words \\ Fig. 2: 305 words \\ Fig. 3: 97 words \\ Fig. 4: 350 words \\ Fig. 5: 229 words
}

The authors will not pay color reproduction charges. 


\begin{abstract}
In this paper, Large Eddy Simulations (LES) have been performed on an ethylene/air piloted turbulent non-premixed sooting jet flame to quantify the importance of aromatic chemistry-turbulence interactions. Aromatic species are of primary importance since their concentrations control directly the soot nucleation rates. In the current work, the chemistry-turbulence interactions for benzene and naphthalene are taken into account by solving transport equations for their mass fractions. A recently developed relaxation model is used to provide closure for their chemical source terms. The effects of turbulent unsteadiness on soot yield and distribution are highlighted by comparing the LES results with a separate LES using tabulated chemistry for all species including the aromatic species. Results from both simulations are compared to experimental measurements. Overall, it is shown that turbulent unsteady effects are of critical importance for the accurate prediction of not only the inception locations, but also the magnitude and fluctuations of soot.
\end{abstract}

Key words: large eddy simulation, soot, turbulent non-premixed flames, chemistry-turbulence interactions

\title{
1 Introduction
}

Due to the detrimental effects of soot emission on human health and the environment, ever more stringent international regulations placed on industrial combustion system emissions make the design of cleaner and more efficient combustion devices a necessity. Towards this end, substantial research efforts are devoted to the numerical prediction of soot formation in turbulent reacting flows [1-3]. Soot nucleates from Polycyclic Aromatic Hydrocarbons (PAH), which involve complex chemical kinetics $[4,5]$. Incorporating such a detailed 
chemical mechanism containing hundreds of species and thousands of reactions into three-dimensional turbulent flow simulations using a finite-rate chemistry model is limited by the current capability of computing power.

In this context, tabulated chemistry based on the non-premixed flamelet model $[6,7]$ is an attractive alternative to finite-rate chemistry, and has been a popular modeling approach to simulate numerically turbulent flames [8-12]. The distinct advantage offered by the flamelet model, compared to finite-rate chemistry, is that flow properties and chemical kinetics are essentially decoupled [13]. More specifically, flamelet equations are solved in advance to build flamelet libraries, from which species mass fractions, temperature, and other thermochemical properties are evaluated in the flow simulations.

One substantial simplification made by the flamelet model is that the characteristic chemical time scales are much smaller than those of turbulence. In other words, chemistry is assumed to respond infinitely fast to turbulent perturbations. Such assumption has been shown to be valid for the major chemical species (reactants and products) as well as radicals (H, OH, O, etc.) [14]. However, due to the large time scales characterizing the PAH chemistry, transient effects could be substantial $[14,15]$. Previous studies have shown that the concentrations of PAH in turbulent non-premixed flames deviate from those predicted by the flamelet model [1]. These observed differences are believed to be a consequence of the rapidly changing turbulent flow field and the slow adjustment of PAH chemistry [1]. Based on the above considerations, unsteady turbulence-chemistry interaction needs to be properly treated for PAH molecules.

Transient effects for PAH molecules have been included first in Large Eddy Simulations (LES) by Mueller and Pitsch [15]. They proposed to solve a trans- 
port equation for a lumped PAH variable. The chemical source term of this variable was closed using the same relaxation model as proposed by Ihme and Pitsch [16] for $\mathrm{NO}_{\mathrm{x}}$, which relies on a reversible one-step global reaction. Only the unsteady evolution of the chemical consumption rate of $\mathrm{PAH}$ was taken into account [15]. Recently, a conceptually similar, but physically more reliable relaxation model for aromatic species has been proposed and validated based on the chemical response of the unsteady flamelet equations to turbulent perturbations [14]. This new model relies on a multi-step process and distinguishes between major PAH species. The unsteady evolution of both chemical consumption and production terms is included.

Although chemistry-turbulence interactions for aromatic species have already been included in LES, their effects and importance have never been investigated. The objective of this work is to investigate the effects of the aromatic chemistry-turbulence interactions on the formation and later evolution of PAH and soot. Particular attention is paid to the inception locations, magnitude and fluctuations of aromatic species and soot.

This paper is organized as follows. Section 2 describes the models used for combustion, LES closure, PAH, and soot transport. In Section 3 and 4, the presented models are applied to the LES of an ethylene/air turbulent nonpremixed jet flame.

\section{$2 \quad$ Numerical algorithms}

The proposed simulation framework relies on four major components: a gasphase chemistry model, a turbulence closure model, a PAH relaxation model, and finally a soot model. 


\subsection{Gas-phase chemistry model}

In the current work, the combustion processes are described using the flamelet/progress variable (FPV) approach [11], extended to include radiative heat losses in the limit of optically thin medium, similar to [16]. The local thermochemical quantities $\phi$ are parametrized using three variables, namely mixture fraction $Z$, progress variable $C$, and an enthalpy loss parameter $H$

$$
\phi=\mathfrak{F}(Z, C, H)
$$

where $C$ is defined as the sum of the mass fractions of $\mathrm{CO}_{2}, \mathrm{CO}, \mathrm{H}_{2} \mathrm{O}$, and $\mathrm{H}_{2}$ [16]. Assuming unity Lewis number $[8,10,17]$, the transport equations for these variables can be written as

$$
\frac{\partial \rho \xi}{\partial t}+\nabla \cdot(\rho \xi \mathbf{u})=\nabla \cdot(\rho D \nabla \xi)+\dot{\omega}_{\xi}
$$

where $\xi$ represents $Z, C$, and $H$. The radiation source term for the enthalpy loss parameter includes contributions from both gas-phase and soot radiation. Gas-phase radiation is evaluated using the RADCAL model [18], and soot radiation is modeled following [19]. The database for chemistry tabulation is generated using solutions to the flamelet equations [20]. Source terms, species mass fractions, and other thermochemical quantities are pre-computed and stored. Further details of the FPV approach can be obtained from $[15,16]$.

\subsection{LES closure}

In this LES, the continuity, momentum, and scalar transport (Eqs. (2)) are filtered spatially. All subfilter stresses and scalar fluxes are closed using a dy- 
namic Smagorinsky model [21-23] with Lagrangian averaging techniques [24,25]. The filtered thermochemical quantities are obtained from the equation of state (Eq. (1)) by convolution with a joint subfilter PDF $\widetilde{P}$

$$
\widetilde{\phi}=\iiint \mathfrak{F}(Z, C, H) \widetilde{P}(Z, C, H) d Z d C d H
$$

Since $Z, C$, and $H$ are not statistically independent variables, a different formulation, equivalent to the above equation, is used in practice

$$
\widetilde{\phi}=\iiint \mathfrak{G}(Z, \Lambda, \Theta) \widetilde{P^{\prime}}(Z, \Lambda, \Theta) d Z d \Lambda d \Theta
$$

with $\Lambda=C\left(Z_{s t}\right)$ and $\Theta=H\left(Z_{s t}\right)$ being mixture-fraction-independent variables. In Eq. $4, \mathfrak{G}(Z, \Lambda, \Theta)$ is a different, yet equivalent, form of the equation of state (Eq. 1) [16]. A presumed PDF approach [26-28] is used for $\widetilde{P^{\prime}}(Z, \Lambda, \Theta)$. A beta distribution is used for mixture fraction, based on its filtered value $\widetilde{Z}$ and variance $\widetilde{Z^{\prime 2}}$; Dirac distributions are used for $\Lambda$ and $\Theta$ [16]. Further, by assuming a unique inversion between $(\widetilde{C}, \widetilde{H})$ and $(\widetilde{\Lambda}, \widetilde{\Theta})[16]$, the filtered thermochemical quantities can be expressed as

$$
\widetilde{\phi}=\widetilde{\mathfrak{L}}\left(\widetilde{Z}, \widetilde{Z^{\prime 2}}, \widetilde{C}, \widetilde{H}\right)
$$

where $\widetilde{\mathfrak{L}}$ is the flamelet library established based on the flamelet solutions, convoluted with the presumed subfilter PDF. The filtered variance of the mixture fraction $\widetilde{Z^{\prime 2}}$ is obtained using an algebraic model [29,30]. 


\subsection{PAH relaxation model}

Soot formation depends critically on the concentrations of its precursors, namely aromatic species, which exhibit substantial transient effects due to turbulent unsteadiness [1]. To account for these effects, transport equations (Eq. (2) spatially filtered) are solved for their mass fractions [15,31]. The chemical source terms for these transported aromatic species are closed using a recently developed relaxation model [14]. The general picture of this relaxation model is depicted in Fig. 1.

[Fig. 1 about here.]

This model, similar to [16], takes into account the unsteady evolution of both the chemical production $\left(\dot{\omega}^{+}\right)$and consumption terms $\left(\dot{\omega}^{-}\right)$. All chemical reactions leading to the formation of an aromatic species are grouped into a representative production reaction, and all chemical reactions leading to its destruction are grouped into a different representative consumption reaction. As a result, the chemical source terms for a PAH with $n$ aromatic rings $\left(A_{n}\right)$ is given by

$$
\dot{\omega}_{A_{n}}=a \cdot Y_{A_{n-1}}-b \cdot Y_{A_{n}}
$$

The coefficients $a$ and $b$ (both positive) were found independent of the species mass fractions [14] and can be tabulated solely using flamelet solutions by dividing the production and consumption rates of $A_{n}$ by the mass fraction of $A_{n-1}$ and $A_{n}$, respectively

$$
a=\frac{\dot{\omega}_{A_{n}}^{+}}{Y_{A_{n-1}}} \text {, and } b=\frac{\dot{\omega}_{A_{n}}^{-}}{Y_{A_{n}}}
$$


The current study presents a simplified case of this general schematic (Fig. 1), since only two aromatic species are considered. Benzene $\left(\mathrm{C}_{6} \mathrm{H}_{6}\right)$ is the first aromatic species formed in the gas-phase mixture. Therefore, in this model, it is the first species for which unsteady effects are considered and its overall chemical source term is tabulated directly based on flamelet solutions. For aromatic molecules beyond benzene, the model represented by Eq. (6) is used. In the current work, as a first step, only benzene and naphthalene $\left(\mathrm{C}_{10} \mathrm{H}_{8}\right)$ are considered, primarily due to the relatively high uncertainties in the chemistry of PAH larger than naphthalene. It is important to note that the accuracy of the proposed PAH relaxation model is limited by that of the full chemical mechanism.

Following the turbulent closure proposed by $[15,16]$, the final form of the filtered chemical source terms for $\mathrm{C}_{6} \mathrm{H}_{6}$ and $\mathrm{C}_{10} \mathrm{H}_{8}$ is given by

$$
\begin{aligned}
\overline{\dot{\omega}}_{\mathrm{C}_{6} \mathrm{H}_{6}} & =\overline{\dot{\omega}}_{\mathrm{C}_{6} \mathrm{H}_{6}}\left(\widetilde{Z}, \widetilde{Z^{\prime 2}}, \widetilde{C}, \widetilde{H}\right), \\
\overline{\dot{\omega}}_{\mathrm{C}_{10} \mathrm{H}_{8}} & =\bar{a}\left(\widetilde{Z}, \widetilde{Z^{\prime 2}}, \widetilde{C}, \widetilde{H}\right) \cdot \widetilde{Y}_{\mathrm{C}_{6} \mathrm{H}_{6}} \\
& -\bar{b}\left(\widetilde{Z}, \widetilde{Z^{\prime 2}}, \widetilde{C}, \widetilde{H}\right) \cdot \widetilde{Y}_{\mathrm{C}_{10} \mathrm{H}_{8}} \\
& -\bar{d}\left(\widetilde{Z}, \widetilde{Z^{\prime 2}}, \widetilde{C}, \widetilde{H}\right) \cdot\left(\widetilde{Y}_{\mathrm{C}_{10} \mathrm{H}_{8}}\right)^{2},
\end{aligned}
$$

in terms of spatially filtered quantities. The third term on the right hand side of Eq. (9) corresponds to the removal of naphthalene due to dimerization, leading to the nucleation of soot. The turbulent closure of this dimerization term, same as introduced by Mueller and Pitsch [15], approximates the average of a square, $\widetilde{Y^{2}} \mathrm{C}_{10} \mathrm{H}_{8}$, by the square of the average, $\left(\widetilde{Y}_{\mathrm{C}_{10} \mathrm{H}_{8}}\right)^{2}$. Therefore, the filtered naphthalene dimerization rate is slightly under-estimated. The present 
work assumes that soot is nucleated only from the dimerization of naphthalene, since it is the largest PAH considered. Transporting larger PAH molecules to nucleate from will be considered in future work.

\subsection{Soot model}

In the present study, the geometry of a fractal soot aggregate is described using a bi-variate description based on its total volume $V$ and total surface area $S[32,33]$. The evolution of soot particles is described, from a statistical point of view, by solving the transport equations for several key moments,

$$
M_{x, y}=\iint n(V, S) V^{x} S^{y} \mathrm{~d} V \mathrm{~d} S
$$

of the soot Number Density Function (NDF) $n(V, S)$. In the current study, the soot NDF is approximated using two delta functions. The first delta function is intended to capture the population of small, young soot particles, and the second delta function corresponds to the population of large, mature soot particles $[34,35]$.

Soot moment transport equations, derived from the population balance equation of the soot NDF, are [36]

$$
\frac{\partial M_{x, y}}{\partial t}+\nabla \cdot\left(\mathbf{u}^{*} M_{x, y}\right)=\dot{\omega}_{x, y}
$$

where

$$
\mathbf{u}^{*}=\mathbf{u}-0.556 \frac{\nu}{T} \nabla T
$$

is the velocity vector accounting for thermophoresis effects $[1,15], \nu$ is fluid kinematic viscosity, and $\dot{\omega}_{x, y}$ accounts for the nucleation, surface growth, co- 
agulation, condensation, and oxidation of soot particles [36]. The source terms, $\dot{\omega}_{x, y}$, are determined using the Direct Quadrature Method of Moments (DQMOM) [32]. Details of the form of the different source terms and their closure can be found in $[15,32,36]$.

The transport equations for four soot moments are spatially filtered,

$$
\begin{aligned}
& \frac{\partial \bar{M}_{x, y}}{\partial t}+\nabla \cdot\left(\widetilde{\mathbf{u}^{*}} \bar{M}_{x, y}\right) \\
= & \nabla \cdot\left(\widetilde{\mathbf{u}^{*}} \bar{M}_{x, y}-\overline{\mathbf{u}}^{*} M_{x, y}\right)+\overline{\dot{\omega}}_{x, y} .
\end{aligned}
$$

The subfilter moment fluxes are modeled using a dynamic Smagorinsky model [21$23]$ with Lagrangian averaging techniques $[24,25]$. For the turbulent closure of the soot moment source terms, the portion which is a function of the thermochemical state of the gas is tabulated as any other gas quantities (Eq. (3)). The other portion is treated using laminar closure [15].

\section{Sandia flame: simulation details}

The described models are integrated into LES of a non-premixed ethylene/air piloted turbulent jet flame, experimentally investigated at Sandia National Laboratories [37-39]. This flame is selected over other experimentally studied turbulent non-premixed flames [40,41], for its relatively large soot yield, high Reynolds number, and well-defined boundary conditions, as described in detail below. 


\subsection{Flame configuration}

The burner consists of two concentric tubes, with high-speed fuel injection in the inner one, and low-speed injection of fuel/air mixture in the outer one (pilot flame). The flame is attached to the burner and stabilized by 64 tiny premixed pilot flames arranged in three concentric rings, which guarantee a flat pilot flow profile. While the fuel jet delivers pure ethylene, premixed ethylene/air mixture at an equivalence ratio of 0.9 is supplied as pilot stream, at a flow rate corresponding to $2 \%$ of the energy release rate of the main jet. Finally, the burner sits on top of a vertical wind tunnel, providing wide coflowing air to prevent room-air disturbances. A more detailed description of the burner configuration is given in [37]. Based on the bulk velocity and diameter of the fuel jet, the jet Reynolds number is estimated to be $R e=20,000$.

\subsection{Numerical set-up}

The filtered conservation equations for mass, momentum, mixture fraction, progress variable, enthalpy loss parameter, the two aromatic species mass fractions (benzene and naphthalene), and four soot moments are solved in a cylindrical coordinate system using the NGA code [42]. The scalar equations are discretized spatially using the BQUICK scheme [43]. This scheme ensures that the physical bounds of appropriate quantities are numerically preserved throughout the simulation without adding significant artificial diffusion. The flames have been computed for more than 10 flow through times.

Based on the visible flame height and width measured in the experiments, the size of the computational domain is selected to be $1 m \times 0.3 m \times 2 \pi$ in 
the axial, radial, and azimuthal directions, respectively. The grid in the axial direction uses 384 points and is stretched downstream from the burner exit. The radial direction is discretized using 204 non-uniformly distributed grid points concentrated in the shear layers between the different injection streams. The azimuthal direction is equally spaced using 64 grid points. The turbulent inlet velocity profile for the fuel stream is extracted from a separate, fullydeveloped periodic pipe flow simulation with the experimentally measured axial mean bulk velocity.

For the combustion model, the flamelet equations are solved using the FlameMaster code [44]. The resulting flamelet library leads to a smooth mapping of all the branches of the S-shaped curve. It is discretized with $100 \times 25 \times 100 \times 100$ grid points in $\widetilde{Z}, \widetilde{Z^{\prime 2}}, \widetilde{C}$, and $\widetilde{H}$ directions, respectively. The detailed chemical mechanism employed in the present flamelet calculations was initially developed by Blanquart et al. $[5,45]$, with slight modifications made since its first publication. The chemical mechanism can be found in the supplemental material of [14]. It contains 168 species and 1878 reactions (forward and backward reactions counted separately) and takes into account all major pathways of PAH formation. This chemical model has been extensively tested and validated for PAH and soot formation in multiple configurations, which guarantees the accuracy of the resulting PAH relaxation model $[5,45]$.

\section{Results and discussion}

\subsection{Instantaneous fields}

[Fig. 2 about here.] 
The instantaneous fields of temperature, benzene mass fraction, naphthalene mass fraction, and soot volume fraction are depicted in Fig. 2.

As expected, the yield of aromatic species and soot is predominant in fuel rich regions inside the stoichiometric mixture fraction iso-contour.

The formation of benzene is shown to initiate at $x / d \simeq 15$, while naphthalene is observed to form only after $x / d \simeq 30$. Soot formation is very intermittent, and the location where soot forms may appear different from one time instant to another. However, soot formation occurs generally at more downstream locations at $x / d \simeq 50$ (Fig. 3). These lags in formation locations reflect the combined effects of the sequential formation of aromatic species and soot, and the large time scales governing their formation. Soot volume fraction and naphthalene mass fraction are found to be highly intermittent, where thin and confined regions of high soot volume fraction are followed by extended low soot volume fraction regions [1], similarly to the experimental observations [37].

\subsection{Mean soot profile}

To highlight the effects of the interactions between turbulence and PAH chemistry on soot, a separate LES is performed, in which aromatic species concentrations are tabulated directly, as for all other gas-phase species. In the following discussion, this second simulation is referred to as "steady-state LES", while the original simulation (aromatic species being transported) is referred to as "relaxation LES". The two LES have been performed keeping everything else constant; the only difference is the use of the relaxation model in the relaxation LES. For this reason, differences between the results from both simulations can be attributed to the effects of PAH relaxation. 
[Fig. 3 about here.]

Figure 3 shows the time-averaged soot volume fractions on the flame centerline from both LES and from laser-induced incandescence measurements [39]. The relative uncertainty of the experimental data was $\pm 20 \%$ [39]. Comparison between LES results and experimental data indicates that the relaxation LES performs better than the steady-state LES and that using the relaxation model leads to encouraging agreement with experimental measurements. The relaxation LES predicts the correct location of soot inception around $x / d=50$, where the gradual increase in soot volume fraction through inception and growth is also qualitatively well reproduced. The well-predicted soot inception behavior in the relaxation LES and the pre-mature soot inception predicted by the steady-state LES can be observed more clearly in Fig. 4(b). The peak in soot volume fraction occurs close to $x / d=150$ in both experiments and the relaxation LES. At this location, the optical depth was calculated to be around $0.02(\ll 1)$ through a flame radius. The optically thin assumption made in Section 2.1 is therefore acceptable. The magnitude of the mean soot volume fraction is slightly under-predicted for $x / d<180$. There are many reasons for the lower soot mean concentration, for instance the under-estimated filtered naphthalene dimerization rate (Eq. (9)) and the exclusion of PAH species larger than naphthalene in the present study. Including more species to nucleate from would increase the total soot yield. At $x / d>180$, oxidation dominates and eliminates soot. The relatively satisfactory mean volume fraction prediction at these locations is primarily due to the good prediction of the flame height, and indicates that the oxidation process is also well captured.

On the other hand, the steady-state LES predicts a lower soot yield and a slightly shifted soot profile towards the burner exit (peak in soot volume frac- 
tion occurs around $x / d=135)$. Furthermore, the increase in soot volume fraction, depicting the soot inception processes, starts closer to the burner exit (see also Fig. 4(b)). This pre-mature soot nucleation is not surprising as the yield of aromatic species is already at the flamelet-predicted steady-state values at the burner exit.

\subsection{Effects of PAH chemistry-turbulence interaction}

[Fig. 4 about here.]

In Fig. 4, time-averaged fields obtained from the relaxation LES are compared against those obtained from the steady-state LES. Several observations can be made.

First of all, a systematic lag in the formation locations of naphthalene and soot is observed between the two simulations. The aromatic species (benzene not shown) and soot are found to form at locations closer to the burner exit in the steady-state LES. Their delayed formation in the relaxation LES is due to the interactions between turbulence and their slow chemistry. It is interesting to note that the locations at which soot volume fraction peak are very close between these $\operatorname{LES}(x / d \simeq 135$ v.s. $x / d \simeq 150)$, despite the differences in inception locations (also shown in Fig. 3). This is primarily because that the location where soot peaks corresponds to where soot nucleation balances soot oxidation. Since the height of the flame differs only by $6 \%$ in the two LES, the peak soot locations in the two LES are roughly the same.

Second, taking into account these unsteady effects (by transporting aromatic species) leads to larger aromatic species concentrations. The maximum mean 
mass fraction of benzene is around 1.6 times larger in the relaxation LES than in the steady-state LES. A similar observation can be made for naphthalene. The maximum mean soot volume fractions found in the relaxation LES and steady-state LES are $0.36 \mathrm{ppm}$ and $0.15 \mathrm{ppm}$, respectively. This factor of 2.4 difference in soot is consistent with the factor of 1.5 difference observed in naphthalene, since the dimerization rate leading to soot nucleation scales as the naphthalene mass fraction to the second power (Eq. (9)). The higher predicted benzene, naphthalene, and hence soot yield in the relaxation LES, can be explained by the non-monotonic time-evolution of aromatic species [14].

Finally, lower temperatures are found in the relaxation LES, compared to the steady-state LES. These differences $(\simeq 100 K)$ are found at the same locations where large soot volume fraction is observed. They are due to the more pronounced radiative heat losses captured in the relaxation LES, associated with a larger predicted soot yield. Consequently, the relaxation LES predicts a flame $15 \%$ longer than that predicted by the steady-state LES $[37,46,47]$.

\subsection{Soot volume fraction fluctuations}

[Fig. 5 about here.]

Chemistry-turbulence interactions impact not only mean quantities, but also the fluctuations in soot, as shown in Fig. 5. Probability Density Functions $(\mathrm{PDF})$ of soot volume fraction are calculated at mid-height $(x / d=140)$ and at an elevated height $(x / d=200)$ on the flame centerline, and they are compared against experimental measurements $[38,38]$. The experimental data are obtained from extinction measurements using the 3-line diagnostic [37,38], with a relative uncertainty of $\pm 12 \%[38,48]$. At these two locations, the size 
of the computational cell is around $3 \mathrm{~mm}$ in the axial direction and $0.5 \mathrm{~mm}$ in the radial direction. The much finer grid size compared to the probe size for extinction measurements $(10 \mathrm{~mm})$ [38] ensures that the comparison between the simulation results and the experimental data is appropriate.

At $x / d=140$, both the mean value and the magnitude of fluctuations (width of the PDF) are well captured by the relaxation LES; although occurrence of low soot volume fractions $\left(f_{v}<0.2 \mathrm{ppm}\right)$ is slightly over-predicted. In contrast, the steady-state LES predicts a lower mean, as aforementioned, and significantly smaller fluctuations (narrower PDF).

At $x / d=200$, the steady-state LES still over-predicts the occurrence of low soot volume fractions. The experimentally observed peak in soot volume fraction PDF centering at $f_{v} \simeq 0.2 \mathrm{ppm}$ is not well captured. It also fails to predict the tail in the experimentally measured PDF at large soot volume fractions: no occurrence of $f_{v}>0.4 \mathrm{ppm}$ is predicted. In the relaxation LES, the tail of the PDF is much better reproduced, since larger fluctuations are enabled by taking into account chemistry-turbulence interactions.

\section{Conclusions}

This paper investigates the effects of turbulent unsteadiness on the formation of aromatic species and soot. Large-eddy simulations have been performed on a non-premixed ethylene/air piloted turbulent jet flame. Transport equations for benzene and naphthalene mass fractions are solved along with the flow field to capture aromatic chemistry-turbulence interactions. Simulation results have shown that, first, these interactions lead to a delayed inception of aromatic species away from the burner exit. The resulting inception locations for soot 
are shown to be in good agreement with experimental measurements. Second, aromatic chemistry-turbulence interactions are also important to reproduce correctly the soot yield in the flame. Neglecting these interactions (steadystate LES) leads to the under-prediction of the maximum mean soot volume fraction by a factor of three. Finally, turbulent unsteadiness is shown to be critical to capture the strong fluctuations in soot volume fraction, as observed in the experiments. Significant under-prediction of the occurrence of high soot volume fractions are shown when chemistry-turbulence interactions are not included for aromatic species.

\section{Acknowledgments}

The authors gratefully acknowledge funding from the U.S. Department of Energy-Basic Energy Sciences (DE-SC006591). The authors deeply acknowledge Dr. Shaddix at Sandia National Laboratories and Dr. Mueller at Princeton University for their valuable comments and fruitful discussions.

\section{References}

[1] F. Bisetti, G. Blanquart, M. E. Mueller, H. Pitsch, Combustion and Flame 159 (1) (2012) $317-335$.

[2] D. O. Lignell, J. H. Chen, P. J. Smith, T. Lu, C. K. Law, Combustion and Flame 151 (12) (2007) 2 - 28.

[3] Yunardi, R. Woolley, M. Fairweather, Combustion and Flame 152 (3) (2008) $360-376$.

[4] H. Wang, M. Frenklach, Combustion and Flame 110 (12) (1997) 173 - 221. 
[5] G. Blanquart, P. Pepiot-Desjardins, H. Pitsch, Combustion and Flame 156 (3) (2009) 588-607.

[6] N. Peters, Combust. Sci. Technol 30 (1) (1983) 1-17.

[7] N. Peters, Prog. Energy combust. Sci 2180 (910) (1984) 319-339.

[8] A. W. Cook, J. J. Riley, Combustion and Flame 112 (4) (1998) 593 - 606.

[9] A. Kempf, A. Sadiki, J. Janicka, Proceedings of the Combustion Institute 29 (2) (2002) $1979-1985$.

[10] H. Pitsch, H. Steiner, Proceedings of the Combustion Institute 28 (1) (2000) 41 -49 .

[11] C. D. Pierce, P. Moin, Journal of Fluid Mechanics 504 (2004) 73-97.

[12] V. Raman, H. Pitsch, R. O. Fox, Combustion and Flame 143 (12) (2005) 56 78.

[13] H. Pitsch, Annu. Rev. Fluid Mech. 38 (2006) 453-482.

[14] X. Yuan, G. Blanquart, Combustion and Flame (2013) in press.

[15] M. E. Mueller, H. Pitsch, Combustion and Flame 159 (6) (2012) 2166-2180.

[16] M. Ihme, H. Pitsch, Physics of Fluids 20 (055110).

[17] R. Barlow, J. Frank, A. Karpetis, J.-Y. Chen, Combustion and Flame 143 (4) (2005) $433-449$.

[18] R. Barlow, A. Karpetis, J. Frank, J.-Y. Chen, Combustion and Flame 127 (3) (2001) $2102-2118$.

[19] G. L. Hubbard, C. L. Tien, Journal of Heat Transfer 100 (1978) 235.

[20] H. Pitsch, N. Peters, Combustion and Flame 2180 (98) (1998) 26-40.

[21] M. Germano, U. Piomelli, P. Moin, W. H. Cabot, Physics of Fluids A: Fluid Dynamics 3 (7) (1991) 1760-1765. 
[22] P. Moin, K. Squires, W. Cabot, S. Lee, Physics of Fluids A: Fluid Dynamics 3 (11) (1991) 2746-2757.

[23] D. K. Lilly, Physics of Fluids A: Fluid Dynamics 4 (3) (1992) 633-635.

[24] C. Meneveau, T. S. Lund, W. H. Cabot, Journal of Fluid Mechanics 319 (1996) $353-385$.

[25] J. Reveillon, L. Vervisch, Physics of Fluids 8 (8) (1996) 2248-2250.

[26] A. W. Cook, J. J. Riley, Physics of Fluids 6 (8) (1994) 2868-2870.

[27] J. Jimenez, A. Linan, M. M. Rogers, F. J. Higuera, Journal of Fluid Mechanics 349 (1997) 149-171.

[28] C. Wall, B. J. Boersma, P. Moin, Physics of Fluids 12 (10) (2000) 2522-2529.

[29] C. D. Pierce, P. Moin, Physics of Fluids 10 (12) (1998) 3041-3044.

[30] G. Balarac, H. Pitsch, V. Raman, Physics of Fluids 20 (3).

[31] P. Donde, V. Raman, M. E. Mueller, H. Pitsch, Proceedings of the Combustion Institute 34 (1) (2013) 1183 - 1192.

[32] G. Blanquart, H. Pitsch, Combustion and Flame 156 (8) (2009) 1614-1626.

[33] M. E. Mueller, G. Blanquart, H. Pitsch, Proceedings of the Combustion Institute 32 (1) (2009) $785-792$.

[34] B. Oktem, M. P. Tolocka, B. Zhao, H. Wang, M. V. Johnston, Combustion and Flame 142 (4) (2005) $364-373$.

[35] A. D. Abid, N. Heinz, E. D. Tolmachoff, D. J. Phares, C. S. Campbell, H. Wang, Combustion and Flame 154 (4) (2008) 775 - 788.

[36] G. Blanquart, H. Pitsch, A joint volume-surface-hydrogen multi-variate model for soot formation, in: H. Bockhorn, A. DAnna, A.F. Sarofim, H. Wang (Eds.), Karlsruhe University Press, Karlsruhe, 2008. 
[37] J. Zhang, C. R. Shaddix, R. W. Schefer, Review of Scientific Instruments 82 (7) (2011) 074101.

[38] C. R. Shaddix, J. Zhang, Proceedings of the 8th U.S. Combustion Meeting (2013).

[39] C. R. Shaddix, J. Zhang, W. Scheffer, J. Doom, J. C. Oefelein, S. Kook, L. M. Pickett, H. Wang, Sandia National Laboratories Report, Sand2010-7178.

[40] T. Peeters, P. Stroomer, J. de Vries, D. Roekaerts, C. Hoogendoorn, Symposium (International) on Combustion 25 (1) (1994) 1241 - 1248.

[41] R. Barlow, J. Frank, Symposium (International) on Combustion 27 (1) (1998) $1087-1095$.

[42] O. Desjardins, G. Blanquart, G. Balarac, H. Pitsch, Journal of Computational Physics 227 (15) (2008) 7125-7159.

[43] M. Herrmann, G. Blanquart, V. Raman, AIAA Journal 44 (12) (2006) 28792886.

[44] H. Pitsch, Technical Report University of Technology (RWTH), Aachen, 1998.

[45] K. Narayanaswamy, G. Blanquart, H. Pitsch, Combustion and Flame 157 (10) (2010) 1879-1898.

[46] M. Delichatsios, Combustion and Flame 92 (4) (1993) 349 - 364.

[47] S. R. Turns, An Introduction to Combustion: Concepts and Applications, McGraw-Hill, Boston, 2000.

[48] J. Murphy, C. Shaddix, Sandia National Laboratories Report, SAND2003-8246. 


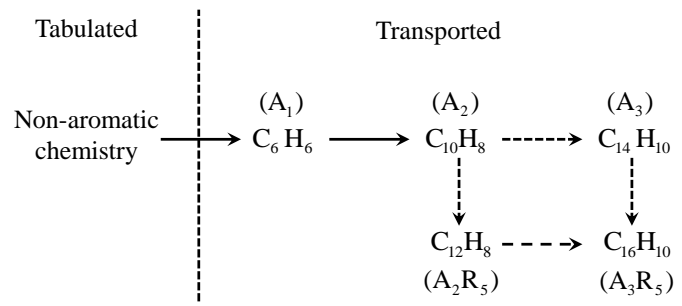

Fig. 1. General diagram of reactions leading to the formation of PAH species in the relaxation model. 


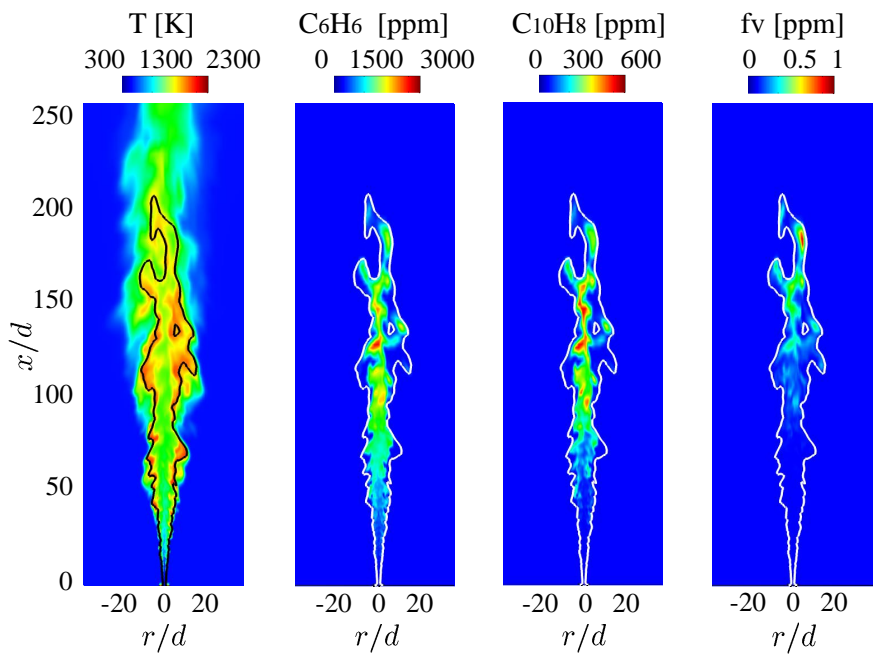

Fig. 2. Instantaneous fields of temperature, benzene mass fraction, naphthalene mass fraction, and soot volume fraction. The iso-contour of stoichiometric mixture fraction (indicating the flame front) is shown in solid line. 


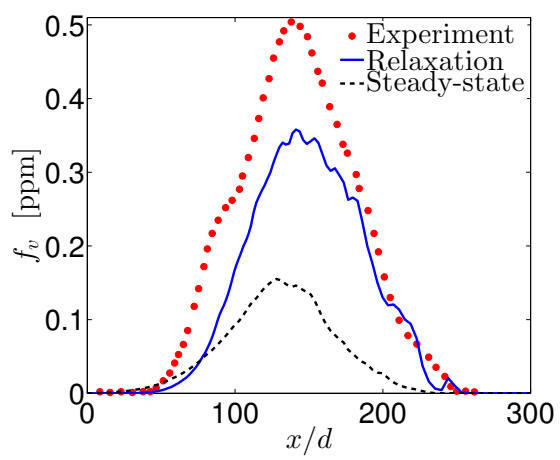

Fig. 3. Mean soot volume fraction on the flame centerline. 


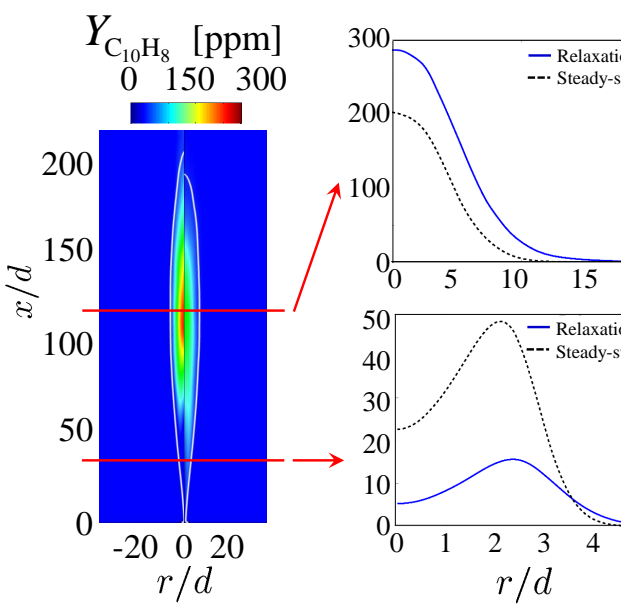

(a) Naphthalene.

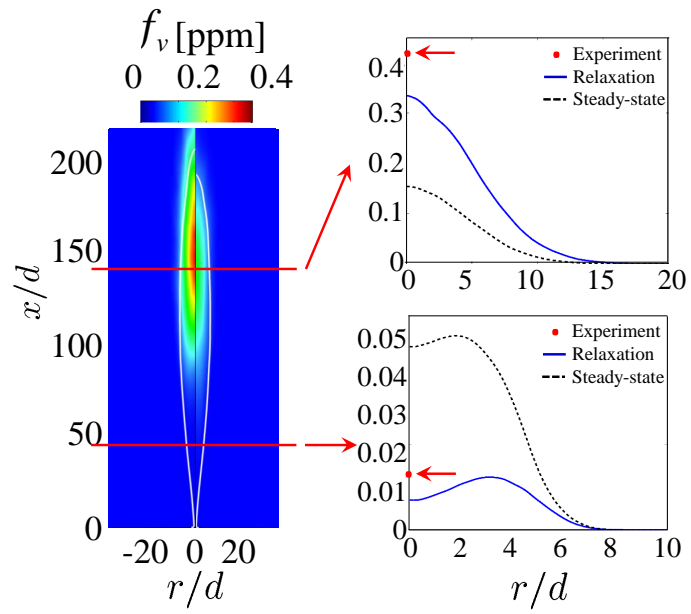

(b) Soot volume fraction.

Fig. 4. Time-averaged fields of naphthalene mass fraction and soot volume fraction. Results obtained using the relaxation model for transported aromatic species are shown on the left half. Results obtained using tabulated aromatic species concentrations are shown on the right half. The iso-contour of stoichiometric time-averaged mixture fraction (indicating the flame front) is plotted in white solid line. Radial profiles are plotted at $x / d=30$ and $x / d=120$ for naphthalene mass fraction, and are plotted at $x / d=40$ and $x / d=140$ for soot volume fraction. Experimental data is highlighted by red arrows. 


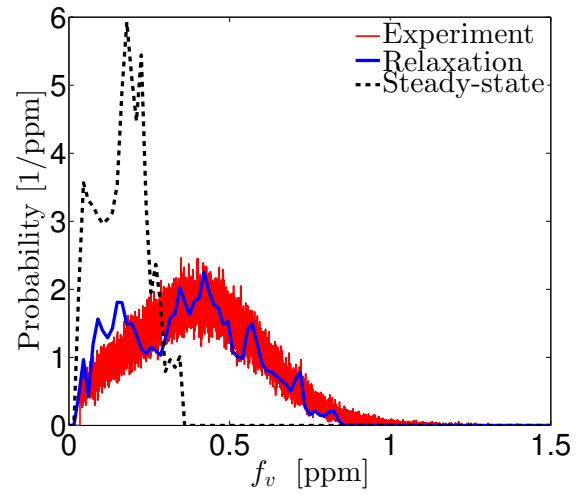

(a) $x / d=140(44.8 \mathrm{~cm})$.

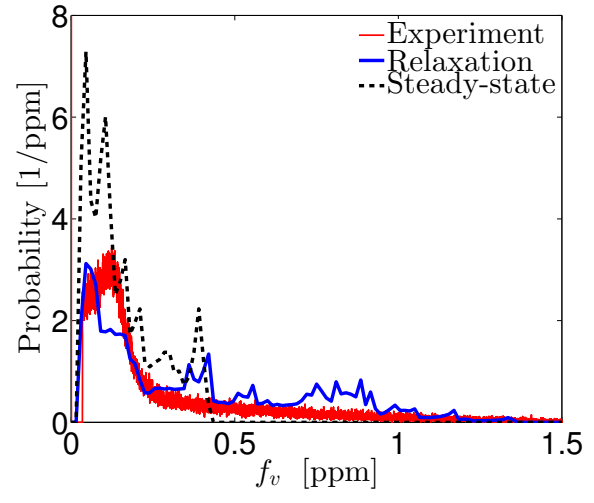

(b) $x / d=200(64.0 \mathrm{~cm})$.

Fig. 5. PDFs of soot volume fraction at two locations on the flame centerline. 


\section{List of Figures}

1 General diagram of reactions leading to the formation of PAH species in the relaxation model.

2 Instantaneous fields of temperature, benzene mass fraction, naphthalene mass fraction, and soot volume fraction. The iso-contour of stoichiometric mixture fraction (indicating the flame front) is shown in solid line.

3 Mean soot volume fraction on the flame centerline.

4 Time-averaged fields of naphthalene mass fraction and soot volume fraction. Results obtained using the relaxation model for transported aromatic species are shown on the left half. Results obtained using tabulated aromatic species concentrations are shown on the right half. The iso-contour of stoichiometric time-averaged mixture fraction (indicating the flame front) is plotted in white solid line. Radial profiles are plotted at $x / d=30$ and $x / d=120$ for naphthalene mass fraction, and are plotted at $x / d=40$ and $x / d=140$ for soot volume fraction. Experimental data is highlighted by red arrows.

5 PDFs of soot volume fraction at two locations on the flame centerline. 